\title{
Optimal Gap Navigation for a Disc Robot
}

\author{
Rigoberto Lopez-Padilla, Rafael Murrieta-Cid and Steven M. LaValle
}

\begin{abstract}
This paper considers the problem of globally optimal navigation with respect to Euclidean distance for a disc-shaped, differential-drive robot placed into an unknown, simply connected planar region with piecewise-analytic boundary. The robot is unable to build precise geometric maps or localize itself in any Euclidean frame. Most of the robot's information comes from a gap sensor, which indicates depth discontinuities and allows the robot to move toward them. A motion strategy is presented that optimally navigates the robot to any landmark in the region. Optimality is proved and the method is illustrated in simulation.
\end{abstract}

\section{Introduction}

If a point robot is placed into a given polygonal region, then computing shortest paths is straightforward. The most common approach is to compute a visibility graph that includes only bitangent edges, which is accomplished in $O\left(n^{2} \lg n\right)$ time by a radial sweeping algorithm [4] (an $(n \lg n+m)$ algorithm also exists, in which $m$ is the number of bitangents [7]). An alternative is the continuous Dijkstra method, which combinatorially propagates a wavefront through the region and determines the shortest path in $O(n \lg n)$ time. Numerous problem variations exist. Computing shortest paths in three-dimensional polyhedral regions is NP-hard [1]. Allowing costs to vary over regions considerably complicates the problem $[14,16]$. See $[6,13]$

Rigoberto Lopez-Padilla

Center for Mathematical Research (CIMAT), Guanajuato, Gto. 36240, México e-mail: rigolpz@cimat.mx

Rafael Murrieta-Cid

Center for Mathematical Research (CIMAT), Guanajuato, Gto. 36240, México e-mail: murrieta@cimat.mx

Steven M. LaValle

University of Illinois, Urbana, IL 61801, USA e-mail: lavalle@uiuc.edu 


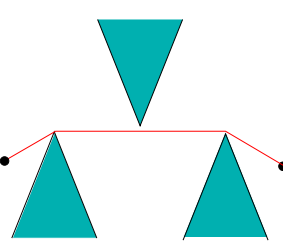

(a)

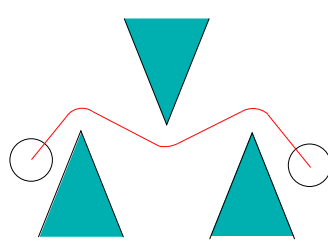

(b)

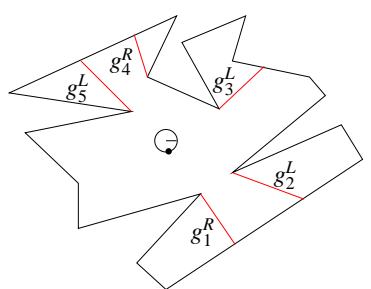

(c)

Fig. 1 a) The optimal path for a point robot, b) The optimal path for a disc robot c) The gap sensor (attached at the small solid disc on the robot boundary) detects the sequence of gaps $G=$ $\left[g_{1}^{R}, g_{2}^{L}, g_{3}^{L}, g_{4}^{R}, g_{5}^{L}\right]$, in which $g_{1}^{R}$ and $g_{4}^{R}$ are near-to-far gaps and $g_{2}^{L}, g_{3}^{L}$, and $g_{5}^{L}$ are far-to-near gaps.

for surveys of shortest path algorithms. For recent efforts on curved obstacles, see [2].

The approaches described thus far address a point robot, which is unrealistic in most practical settings. It is therefore interesting to study the case of a disc robot, which could correspond, for example, to a Roomba platform. Various objective functions are possible; we choose to optimize the distance traveled by the center of the robot. Once the robot has nontrivial dimensions, the problem can be expressed in terms of configuration space obstacles. Solutions are presented in $[3,11]$.

Now suppose that the map of the environment is not given to the robot. It must use its sensors to explore and map the environment to develop navigation strategies. Given strong sensors and good odometry, standard SLAM approaches [5, 17] could be applied to obtain a map that can be used as input to the previously mentioned methods.

However, we do not allow the robot to localize itself or to build a geometric map. Instead, it observes the world using mainly a gap sensor, introduced in [18], which allows it to determine the directions of discontinuities in depth (distance to the boundary) and move toward any one of those directions. Under this model, but for a point robot, a combinatorial filter called the Gap Navigation Tree (GNT) was introduced that encodes precisely the part of the shortest-path visibility graph that is needed for optimal navigation [18]. The learned data structure corresponds exactly to the shortest path tree [6] from the robot's location. This enables the robot to navigate to any previously seen landmark by following the distance-optimal path, even though it cannot directly measure distances. The GNT was extended and applied to exploration in [15]. The GNT was extended to point cloud models in [8]. A larger family of gap sensors is described in [10].

The case of a disc robot is important because real robots have nonzero width. Unfortunately, the problem is considerably more challenging because without additional sensing information, the robot could accidentally strike obstacles that poke into its swept region as it moves along a bitangent. See Figure 1 (a) and (b). The robot must instead execute small detours from the bitangent. Sensing, characterizing, and optimally navigating around these obstructions is the main difficulty of this paper. 
Before proceeding to the detailed model, motion strategy, and case-by-case analysis, several points are important to keep in mind:

1. The robot is placed into an environment, but it is not given the obstacle locations or its own location and orientation. The purpose is to show how optimal navigation is surprisingly possible without sensing this information (i.e., without ordinary SLAM).

2. The robot first learns the GNT by executing a learning phase, which is described in $[9,18]$, and remains unchanged in this paper. The process involves iteratively chasing "unknown" gaps, causing each to split or disappear. Eventually, only primitive gaps, which were formed by appearances of gaps (due to inflection ray crossings), and gaps formed by merging primitives remain. This corresponds to learning the entire shortest-path graph.

3. A simple navigation strategy is provided that guides the robot to any landmark placed in the environment by using the learned GNT. We give precise conditions under which the motions are optimal and prove this statement using case-by-case analysis.

4. We believe that even when the optimality conditions are not met, the strategy itself is close to optimal. Therefore, it may be useful in many practical settings to efficiently navigate robots with simple sensor feedback.

Section 2 formally describes the robot model and the sensor-based motion primitives. Section 3 introduces an automaton that characterizes all possible sequences of motion primitives that could occur when executing optimal motions to a landmark. Section 4 describes how obstacle blockages are detected and handled when the robot chases a gap. This includes detours (that is, the modification of the path encoded in the GNT for a point robot) needed to achieve optimal navigation. Section 5 argues the optimality of the motion strategy. Section 6 presents an implementation in simulation, and Section 7 concludes the paper.

\section{Problem statement}

The robot is modeled as a disc with radius $r$ moving in an unknown environment, which could be any compact set $E \subset \mathbb{R}^{2}$ for which the interior of $E$ is simply connected and the boundary, $\partial E$, of $E$ is the image of a piecewise-analytic closed curve. Furthermore, it assumed that the collision-free subset of the robot's configuration space $\mathscr{C}$ is connected. $\mathscr{C}$-space obstacle corresponds to that of a translating disc, that is, the extended boundary of $E$ which is due to the robot radius ${ }^{1}$.

\footnotetext{
${ }^{1}$ Note that this is the configuration space for a translating disc rather than for a rigid body because of rotational symmetry.
} 


\subsection{Sensing capabilities}

1) Gap sensor: The robot has an omnidirectional gap sensor $[10,18]$, which is able to detect and track two types of discontinuities in depth information: discontinuities from far to near and discontinuities from near to far (in the counterclockwise direction along $\partial E$ ). Figure 1(c) shows a robot in an environment in which the gap sensor detects some near-to-far and far-to-near gaps.

Let $G=\left[g_{1}^{t}, \ldots, g_{k}^{t}\right]$ denote the circular sequence of gaps observed by the sensor. Using this notation, $t$ represents the discontinuity type, in which $t=R$ means a discontinuity from near to far (the hidden portion is the right) and $t=L$ means a discontinuity from far to near (the hidden portion is to the left). For example, the gap sensor in Figure 1(c) detects gaps of different types $G=\left[g_{1}^{R}, g_{2}^{L}, g_{3}^{L}, g_{4}^{R}, g_{5}^{L}\right]$.

We place the gap sensor on the robot boundary and define motion primitives that send the robot on collision-free trajectories that possible contact the obstacles (moving along the boundary of the free subset of the configuration space is necessary for most optimal paths). These motion primitives, described in detail in Section 2.2, allow the robot to rotate itself so that it is aligned to move the gap sensor directly toward a desired gap, move forward while chasing a gap, and follow $\partial E$ while the sensor is aligned to a gap.

Imagine that a differential drive robot is used. It is assumed that the gap sensor can be moved to two different fixed positions on the robot boundary: The extremal left and right sides with respect to the forward wheel direction. One way to implement this is with a turret that allows the robot to move the gap sensor from its right side to its left side and vice versa. Figure 3(b) shows the sensor aligned to a near-tofar gap in which the gap sensor is on the right side of the robot. To align the sensor to a far-to-near gap, the robot moves the gap sensor to the left side of the robot.

Finally, let $\Lambda$ be a static disc-shaped landmark in $E$ with the same radius as the robot. A landmark $\Lambda$ is said to be recognized if the complete landmark is visible from the location of the gap sensor.

2) Side sensors: To detect obstacles that obstruct the robot while it chases a gap, our algorithms need to measure distances between the extremal left and right side robot's points along the direction of the robot heading (forward and backward) and the obstacles. Let those particular robot points be left side point $l p$ and right side point $r p$. The particular direction tangent to the robot boundary at $r p$ is called $r t$. The particular direction tangent to the robot boundary at $l p$ is called $l t$ (See Figure 2(a)). Thus, we assume that the omnidirectional sensor is able to measure distance, indeed based on distance the discontinuities can be detected. Let $d_{R}$ be the distance between $r p$ and the obstacles at the particular direction $r t$, and $d_{L}$ the distance between $l p$ and the obstacles at the particular direction $l t$ (See Figure 2(b)). Let $d_{u}$ be the distance between the omnidirectional sensor and the vertex $u_{i}$ that originated the gap $g_{i}$ to be chased (in Figure 2(c) $g_{i}=g_{0}^{R}$ ). Let $d_{T}$ be the distance between the vertex that the robot is touching (either at $r p$ or $l p$ ) and the vertex that originated the goal gap $g_{0}$ (the gap to be chased). This distance is measured using the omnidirectional sensor (see Figure 2(d)). If the particular direction, either $r t$ or $l t$, is pointing to a reflex vertex (a gap is aligned with this direction), then a discontinuity in the sensor 


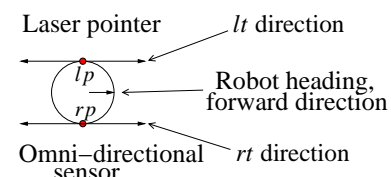

(a)

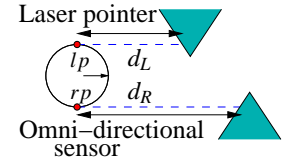

(b)

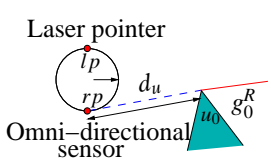

(c)

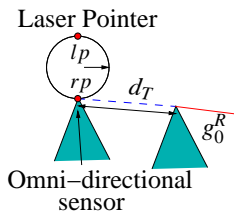

(d)

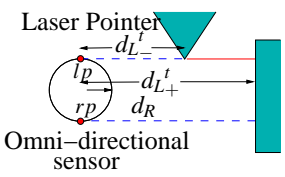

(e)

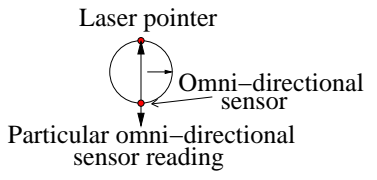

(f)

Fig. 2 Side Sensors: (a) points $r p$ and $l p$, and directions $r t$ and $l t$, (b) $d_{L}$ and $d_{R}$, (c) $d_{u}$, (d) $d_{T}$, (e) $d_{L_{-}}^{t}$ and $d_{L_{+}}^{t}$, (f) omnidirectional sensor readings for contact detection.

reading at this direction occurs. Let $d_{R_{+}}^{t}$ and $d_{R_{-}}^{t}$ denote the distances from $r p$ to the further and closer points, respectively, on $\partial E$ along the discontinuity direction. Similarly, $d_{L_{+}}^{t}$ and $d_{L_{-}}^{t}$ denote the distances from $l p$. See Figure 2(e).

Our motion strategy will require only comparisons of distances to determine which is larger, rather than need precise distance measurements. Any small error in the comparison (if the distances are close) contribute to a small deviation from optimality, which may be relatively harmless in practice. Our approach will furthermore require detecting whether the robot is contacting $\partial E$ at $r p$ or $l p$ to enable wall-following motions.

Distance measurement between the obstacles and $l p$ and $r p$ in directions $r t$ and $l t$ (both backward and forward), and the information of whether the robot is touching $\partial E$ at $r p$ or $l p$, can be obtained with different sensor configurations. For example, it is possible to use two laser pointers and two contact sensor, each of them located at $l r$ and $l p$. However, to use a smaller number of sensors and facilitate the instrumentation of the robotic system, it is possible to emulate both the contact sensors and one of the laser pointers, using the omnidirectional sensor. The omnidirectional sensor reading in the particular forward and backward robot heading directions emulates the laser pointer reading. An omnidirectional sensor can also be used to determine whether the robot is touching $\partial E$ at $l r$ or $l p$. The sensor readings at directions perpendicular to the robot heading are used in this case. If the robot is touching $\partial E$ at the point at which the omnidirectional sensor is located, then the sensor reading is zero. If robot is touching $\partial E$ at the point diametrically opposed to the omnidirectional sensor, then the sensor reading will correspond to robot diameter (see Figure 2(f)). Thus, one option is to have the robot equipped with an omnidirectional sensor and a laser pointer; they will be located at $l p$ and $r p$. Recall that a turret to swap the locations of the laser pointer and the omnidirectional sensor can be used to avoid unnecessary robot rotations in place. The gaps or landmarks are always chased with the omnidirectional sensor; the laser pointer is used to detect obstacles and to correctly align the robot. 


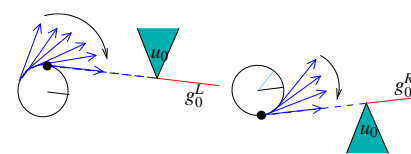

(a)

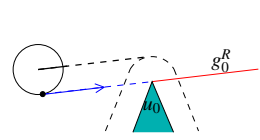

(b)

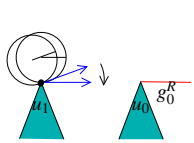

(c)

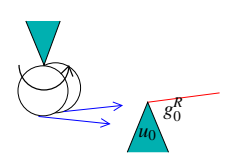

(d)

Fig. 3 The motion primitives: (a) Clockwise rotation in place; (b) Straight line motion; (c) Clockwise rotation w.r.t. to point $r p$; (d) Counterclockwise rotation w.r.t. to point $l p$.

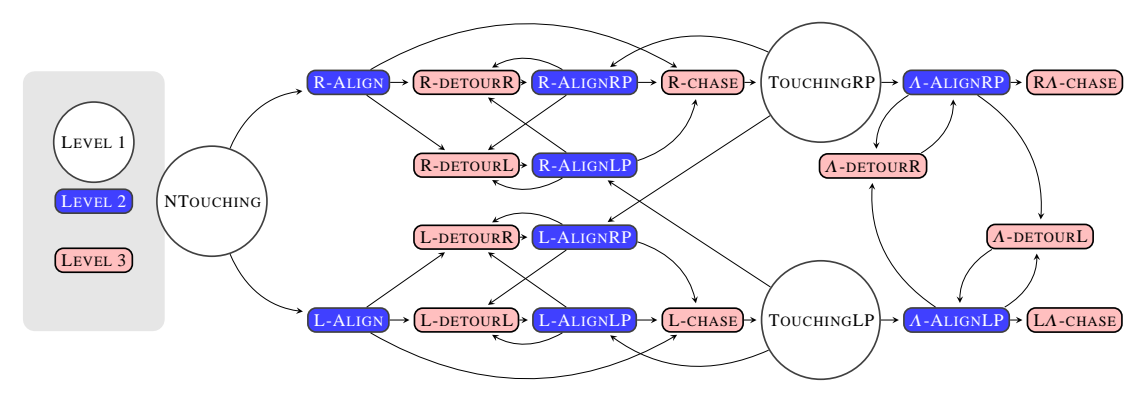

Fig. 4 The sequence of executed primitives depends on sensor feedback. The possible executions are captured by a Moore machine $M$ in which each state applies a specific motion primitive and each transition edge is triggered by a sensing event.

\subsection{Motion primitives}

The robot navigates using a sequence of motion primitives that are generated by an automaton for which state transitions are induced by sensor feedback alone. There are four motion primitives (see Figure 3). Let the angular velocity of the right and left wheels be $w_{r}$ and $w_{l}$, respectively, with $w_{r}, w_{l} \in\{-1,0,1\}$.

Thus, the motion primitives are generated by the following controls:

- Clockwise rotation in place: $w_{r}=1, w_{l}=-1$.

- Clockwise rotation w.r.t. to point $r p: w_{r}=0, w_{l}=1$.

- Counterclockwise rotation w.r.t. to point $l p: w_{r}=1, w_{l}=0$

- Forward straight line motion: $w_{r}=1, w_{l}=1$.

The rotation primitives are used to align $r t$ or $l t$ to a specific gap (or landmark). Once $r t$ or $l t$ is aligned to a gap, the robot moves in straight line to chase the gap. If the path to the chosen gap is blocked, then the robot executes a detour by choosing a new vertex as a subgoal. More details are given in Sections 3 and 4.

\section{The movement automaton}

The algorithm for generating optimal navigation motions can be nicely captured by an automaton or (Moore) finite state machine $M$. See Figure 4. Every state corre- 
sponds to the selection and execution of a motion primitive on the robot or it is a decision. Each state transition is triggered by a sensor feedback event during motion. There are 21 total states, with clear right/left symmetry. The ten upper states in Figure 4 correspond to near-to-far gaps (the $R$ cases) and the ten lower states correspond to far-to-near gaps (the $L$ cases). The other remaining state is NTOUCHING, which is used when the robot is not touching $\partial E$ and decides whether the gap to be chased is left or right.

The machine has 3 main levels (see Figure 4). The first one corresponds to decide whether the goal gap is far-to-near (left gap) o near-to-far (right gap). It also decides whether the robot is touching the $\partial E$. If the robot is touching $\partial E$, then the first level determines whether the robot is touching it at $l p$ (left side) or $r p$ (right side). The second level is the main one, it detects blockages. According to the decisions made in level one, the second level makes the robot executes one of the three types of rotations (clockwise rotation in place, rotation w.r.t. $r p$ or rotation w.r.t. $l p$ ). Before starting the rotation, during robot rotation or after having finished the rotation, the second level determines whether the path to the goal gap is blocked. According to this decision, the robot executes either a straight line motion toward the gap (the path is not blocked) or executes a detour (the robot travels in a straight line toward the subgoal vertex). The third level is in charge of executing the motion toward the gap to be chased.

In the first level no motion primitive is executed. In this level, there are three states:

- NTOuching: This state happens when the robot is not touching $\partial E$. It decides whether the gap to be chased is left of right.

- TouchingRP: This state is triggered when the robot is touching $\partial E$ at $r p$ and the gap being chased splits, or the robot goal is a landmark $\Lambda$ (i.e., the landmark is totally visible to the omnidirectional sensor). The state decides whether the new gap to be chased is a left or right gap. If the new selected gap is a right gap (near-to-far), then the next state will be R-ALIGNRP. If the new selected gap is a left gap (far-to-near), then the next state will be L-ALIGNRP. Finally, whenever the goal is a $\Lambda$ the state will transit to $\Lambda$-ALIGNRP.

- TouchingLP: This state is the left symmetric equivalent to TouchingRP.

The second level determines whether the path to the chosen gap is blocked. To detect the blockage, the robot scans the environment with its sensors and simultaneously aligns either $l t$ or $r t$ to the chased gap $g_{i}$ or the vertex that generates the blockage $u$. This scanning is done by executing three types of rotations (clockwise rotation in place, rotation w.r.t. $r p$ or rotation w.r.t. $l p$ ). There are eight states in the second level (four for the right case and four for the left). The states for the right case are:

- R-Align: Right gap alignment executing clockwise rotation in place.

- R-ALignLP: Right gap alignment executing counterclockwise rotation w.r.t. $l p$.

- R-AlignRP: Right gap alignment executing clockwise rotation w.r.t. $r p$.

- $\Lambda$-AlignRP: Right landmark alignment executing clockwise rotation w.r.t. $r p$. 
Note that whenever the landmark is not reachable by a straight-line path, from the initial robot configuration, then optimal navigation to reach the landmark requires that the landmark is completely visible while the robot is touching $\partial E$.

There are other four other symmetrically equivalent states when the goal gap is left $g_{0}^{L}$, or the landmark is chased with the omnidirectional sensor located at $l p$ (these are $L$ cases in $M$ ).

For all of these states, there are three possible transitions. 1) If the path is not blocked, then a straight line motion is allowed. 2) The robot detects a blockage and the subgoal vertex corresponds to a right gap. 3) There is a blockage and the subgoal vertex generates a left gap.

In the third level, the robot always executes a straight line motion. Either the robot moves to the goal gap $g_{0}$ (these states are called CHASE) or toward the vertex $u$ that represents the subgoal (these states are called DETOUR). Note that the goal vertex or the vertex that blocks the path can generate a left or right gap; for this reason the states are designed as right or left.

There are ten states in the third level (five for the right case and five for the left). The states for the right case are:

- R-CHASE: The robot moves toward the goal gap $g_{0}^{R}$.

- R-DETOURR: The robot moves toward a sub-goal vertex $u_{n}$ that generates a right gap $g_{n}^{R}$ detour.

- R-DETOURL: the robot moves toward a sub-goal vertex $u_{p}$ that generates a left gap $g_{p}^{L}$ detour.

- R $\Lambda$-CHASE: The robot moves toward $\Lambda$, and the omnidirectional sensor is located at $r p$.

- $\Lambda$-DETOURR: The robot moves toward a sub-goal vertex $u_{n}$ that generates a right gap $g_{n}^{R}$ detour to the landmark.

There are five symmetrically equivalent states when the goal gap is left $g_{0}^{L}$ or the landmark is chased with the omnidirectional sensor located at $l p$ (the $L$ cases in $M$ ). This establishes the details of the state machine $M$.

The next section analyzes blockage detection and gap selection. All possible cases for chasing a gap are enumerated. For lack of space, in this paper only two of them are described in detail. An appendix [12] is available online, in which the correctness of the remaining cases is proved.

\section{Blockage detection and gap selection}

In total there are 18 cases to chase a gap; see Figure 5. They can be classified as follows: The first categorization corresponds to a right or left gap, which is the first level in $M$. There are nine cases for each of them. The cases for a left gap $g^{L}$ are completely analogous to the ones for a right gap $g^{R}$. For a right gap, there are three general cases (the second level in Figure 5), and each of them has three subcases (the third level in Figure 5). The second categorization corresponds to the type of 


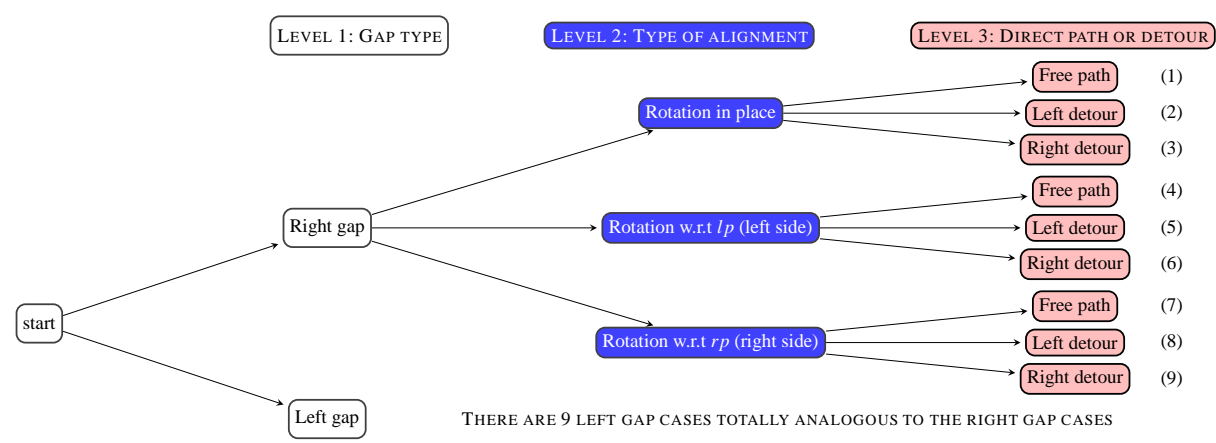

Fig. 5 On the right, nine cases are shown as states in $M$ that are obtained while chasing a gap. Each level corresponds to a critical decision point.

rotation that the robot must execute: Clockwise rotation in place, clockwise rotation w.r.t. $r p$, or counterclockwise rotation w.r.t. $l p$. The rotation is used to scan the environment for detecting a potential blockage and also to correctly align the robot either with the goal gap $g_{0}$ or with the vertex $u$ that represents the subgoal. Note that the vertex that corresponds to the subgoal might generate either a left or right gap. For this reason there are different types of detours. For example, R-DETOURL means that the goal gap is a right gap $g_{0}^{R}$, but a blockage was detected, and the subgoal vertex $u_{p}$ generates a left gap $g_{p}^{L}$. This is the second level in $M$. The third categorization corresponds to the execution of a straight line motion by the robot. Either a detour (left or right) toward the subgoal vertex or a direct path toward the goal gap represents the third categorization, which is the third level in $M$.

Figures 6 (a), (b), and (c) show case 2 in Figure 5. That is, the goal gap is a right gap $g_{0}^{R}$, the robot is not touching $\partial E$, and the vertex $u_{p}$ blocks the path toward the goal gap. The sequence of states to be executed in $M$ is: NTOUCHING, R-ALIGN, R-DETOURL. At the beginning of the task the robot is not touching $\partial E$; hence, $M$ is in state NTOUCHING. The gap to be chased is a right gap denoted $g_{0}^{R}$. The machine $M$ then transitions to R-ALIGN. The first motion primitive executed at state RALIGN is a clockwise rotation in place to align $r t$ with the goal gap $g_{0}^{R}$. At that robot configuration (see Figure 6(a)), the condition to detect the blockage during state RALIGN is: If $d_{R_{-}}{ }^{t}>d_{L}$, then the path toward the right gap $g_{0}^{R}$ is blocked. To correctly align $l t$ with the vertex $u_{p}$ that blocks the path, the robot must execute a clockwise rotation in place until the following condition is satisfied: If direction $l t$ detects a left gap (a discontinuity from far-to-near occurs at direction $l t$ ) and $d_{L_{+}}^{t}>d_{u}$ then the gap $g_{p}^{L}$ is the subgoal gap to be chased (see Figure 6(b)). This finishes R-ALIGN and $M$ transitions to R-DETOURL, which causes a straight line execution of the robot towards $u_{p}$ until it touches the vertex $u_{p}$ at point $l p$.

Figure 6(c) shows that in $E$ the robot has crossed the bitangent line with respect to $\partial E$; however, the corresponding bitangent in the configuration space (of the translating disc) has not been crossed. The figure shows with a red dotted segment, the bitangent line from $u_{p}$ to $u_{0}$. However, in the configuration space, the corresponding bitangent is displaced. In the figure, the bitangent line between $v_{p}$ and $v_{0}$ is 
shown with a black dashed segment. This case appears due to the displacement of a bitangent line due to the robot radius.

Lemma 1. The condition that R-ALIGN uses to detect a left detour is correct, and the new gap selected as goal is the gap that must be chased to obtain locally optimal navigation.

Proof. If $r t$ is aligned with a right goal gap, then the use of distances $d_{R-}^{t}$ and $d_{L}$ is sufficient to detect the blockage, since both distances $d_{R-}^{t}$ and $d_{L}$ are measured at the extremal side robot points in the direction that delimits the area of the robot. Therefore, if $d_{R_{-}}{ }^{t}>d_{L}$ then the path is blocked.

The gap to be chased is the correct gap to obtain locally optimal navigation. Given that the path is blocked the robot executes a clockwise rotation in place to scan the environment and find the sub-goal vertex corresponding to the shortest detour (in the sense of Euclidean distance). During robot rotation, only two different alignment might occur: 1) a left gap is aligned with $l t$ or a right gap is aligned with $r t$. In order to determine the shortest detour, there are only two possibilities: 1) $l t$ is aligned to the previous vertex $u_{p}$ in clockwise order w.r.t the goal vertex $u_{0}$ and $u_{p}$ is closer to the robot than $u_{0} ; u_{p}$ generates a left gap and consequently a left detour. 2) $r t$ is aligned to a next vertex $u_{n}$ in clockwise order w.r.t the goal vertex $u_{0}$ and $u_{n}$ is closer to the robot than $u_{0} ; u_{n}$ generates a right gap and consequently a right detour. Considering $u_{n}$ and $u_{p}$, the vertex that can be reached by a straight line collision-free path is the correct vertex to select as subgoal. Notice that only one vertex, either $u_{n}$ or $u_{p}$ can be reached by a straight line collision-free path.

In this case, during the robot clockwise rotation in place, the alignment of $l t$ with $u_{p}$ occurs before (w.r.t clockwise order) than the alignment of $r t$ with $u_{n}$. Since robot executed a clockwise rotation w.r.t. $r p$ until $l t$ is aligned with $u_{p}$, and at this robot configuration $d_{L-}^{t}<d_{R}$ then a collision free path between that robot configuration and $u_{p}$ exists. Since $d_{L_{+}}^{t}>d_{u}$, then $u_{p}$ is the previous vertex in clockwise order w.r.t the goal vertex $u_{0}$. Since $d_{L-}^{t}<d_{u}$ then $u_{p}$ is closer to the robot than $u_{0}$. Therefore, vertex $u_{p}$ corresponds to the sub-goal yielding the shortest detour toward the goal gap. Consequently, the gap generated by $u_{p}$, a left gap, is the new gap to be chased. The result follows.

Figures 6 (d) and (e) show case 4. The goal gap is a right gap $g_{0}^{R}$, the robot is touching $\partial E$ at $l p$, and there is a straight collision-free path to chase the goal gap $g_{0}^{R}$. The first motion primitive executed is a counter-clockwise rotation w.r.t point $l p$ to align $l t$ with the goal gap $g_{0}^{R}$. Note that at the beginning the omnidirectional sensor was located at point $l p$ because it was chasing the gap related to vertex $u_{T}$, until the gap originated by this vertex split. The turret swap the omnidirectional sensor to point $r p$ and the laser pointer to point $l p$. At that robot configuration (See Fig. 6(d)), the robot starts scanning the environment to detect a blockage. Since there is not a blockage, the robot rotates counterclockwise w.r.t. point $l p$ until $r t$ is aligned to $g_{0}^{R}$. Finally, the robot executes a straight line robot motion toward $g_{0}^{R}$.

Figure 6(e) shows with a (red) doted line, the GNT-encoded path corresponding to a straight line toward the vertex $u_{0}$ (the bitangent line from $u_{T}$ to $u_{0}$ ). In $\mathscr{C}$ there is also a direct path (or bitangent line) between $v_{T}$ and $v_{0}$. 


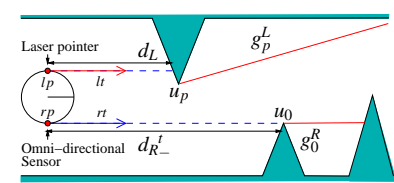

(a)

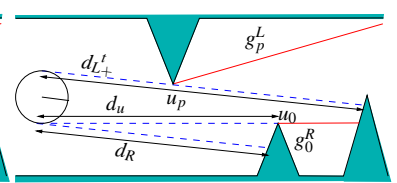

(b)

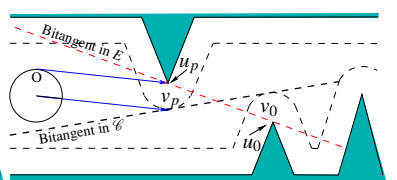

(c)

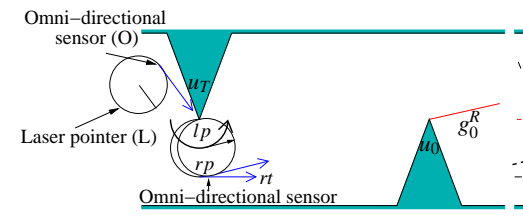

(d)

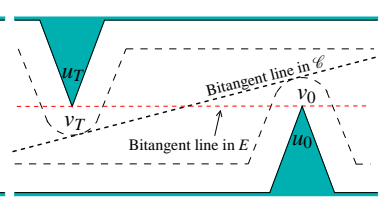

(e)

Fig. 6 Blockage detection and gap selection: Case 2 (a,b,c) and case 4 (d,e). In figures c) and e) the configuration space is shown with doted lines

Lemma 2. The condition that R-ALIGNLP uses to detect a collision free path is correct, and the original goal gap is the correct gap that must be chased to obtain locally optimal navigation.

Proof. Refer to Figure 6 (d) and (e). If $r t$ is aligned to a right goal gap $g_{0}^{R}$, the use of distances $d_{R}{ }^{t}$ and $d_{L}$ is sufficient to detect a straight collision-free path, since $\mathscr{C}$ is connected and hence a path must exist, and both distances $d_{R-}{ }_{-}$and $d_{L}$ are measured at the extremal side robot points in the direction that delimits the area of the robot. Therefore, if $d_{R-}^{t}<d_{L}$ then the path toward the gap is free.

The feedback motion strategy: Although $M$ represents the decision component of the system, the commands to the motors can be implemented by simple sensor feedback. Only four binary sensor observations affect the control: 1) the robot is touching $\partial E$ with the left side (point $l p$ ); (2) it is touching $\partial E$ with the right side (point $r p$ ); (3) the robot is aligned to a gap; and (4) there is a blockage. Depending on the observation state, one of the four different motion primitives will be executed: (1) straight line motion, (2) clockwise rotation in place, (3) clockwise rotation with respect to point $p r$ and (4) counterclockwise rotation with respect to point $l p$. Recall that the angular velocities of the differential-drive wheels yield one of these motion primitives. Hence, the feedback motion strategy can be established by: $\gamma:\{0,1\}^{4} \rightarrow\{-1,0,1\}^{2}$, which the sensor observation vector be denoted as $y_{i}=(r p$, lp,aligned, blockage $)$, to obtain $\gamma\left(y_{i}\right)=\left(w_{r}, w_{l}\right)$. The set of all 16 possible observation vectors can be partitions be letting $x$ denote "any value" to obtain: $y_{1}=(x, x, 1,0), y_{2}=(0,0,0, x), y_{3}=(0,0, x, 1), y_{4}=(x, 1,0, x), y_{5}=(x, 1, x, 1)$, $y_{6}=(1, x, 0, x)$ and $y_{7}=(1, x, x, 1)$. The strategy $\gamma$ can be encoded as

$$
\begin{gathered}
\gamma\left(y_{1}\right)=(1,1) ; \quad \gamma\left(y_{2} \vee y_{3}\right)=(-1,1) \\
\gamma\left(y_{4} \vee y_{5}\right)=(0,1) ; \quad \gamma\left(y_{6} \vee y_{7}\right)=(1,0)
\end{gathered}
$$

, in which $\vee$ means “or". 


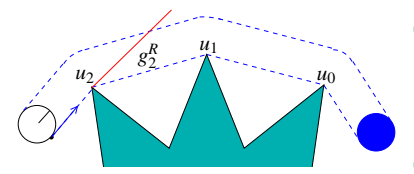

(a)

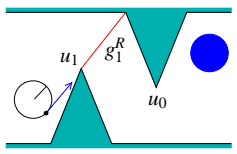

(b)

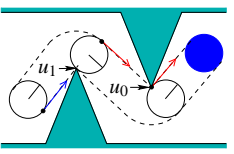

(c)

Fig. 7 a) A non-blocked GNT-encoded path that involves only near-to-far gaps. b) A non-blocked GNT-encoded path that involves both types of gaps (near-to-far and far-to-near). c) Robot path.

\section{Proof of optimal navigation}

\subsection{Non-blocked GNT-encoded paths}

In this section we establish that the robot executes a Euclidean, distance-optimal path in the absence of blockages.

The shortest path to $\Lambda$ is encoded as a sequence of gaps in the GNT. Let $U=\left(u_{n}, u_{n-1}, \ldots, u_{0}\right)$ be the sequence of connected intervals $u_{i} \subset \partial E$ that the robot contacts when the gap sensor (fixed to the robot boundary) moves from its initial position to its final position in $\Lambda$. Let $H=\left(g_{n}, g_{n-1}, \ldots, g_{0}\right)$ denote the corresponding sequence of gaps that are chased, in which $g_{i} \in H$ is the gap that is being chased on the path to $u_{i}$ or while traversing $u_{i}$.

Now consider the problem in terms of the configuration space of the robot. The obstacle region in the configuration space is obtained by growing the environment obstacles by the robot's radius, $r$. Let $\mathscr{C}$ denote the projection of the obstacle region into the plane, thereby ignoring rotation. Let $V=\left(v_{n}, v_{n-1}, \ldots, v_{0}\right)$ be the sequence of intervals $v_{i} \subset \partial \mathscr{C}$ obtained by transforming the interval sequence $U$ from $\partial E$ to $\partial \mathscr{C}$, element by element. The following lemma uses the definition of a generalized bitangent from [18].

Lemma 3. Chasing the sequence $H$ of gaps produces the shortest path if and only if: 1) there is a straight collision-free path from the center of the robot to $v_{n}, 2$ ) there is a (generalized) bitangent line between $v_{i+1}$ and $\left.v_{i}, 3\right)$ there is a straight collision-free path from $v_{0}$ to the landmark center, and 4) $\mathscr{C}$ is connected.

Proof. Note that if any of the first three conditions is violated, then the robot movement is blocked by an obstacle and therefore does not execute an optimal path. For the last condition, if $\mathscr{C}$ is not connected then there is no solution path.

Figure 7(a) shows an example of how $M$ generates an optimal path for the nonblocked case. In the figure, the GNT encodes the sequence $H=\left(g_{2}^{R}, g_{1}^{R}, g_{0}^{R}\right)$. In this example, the machine $M$ traverses the following sequence of states while generating the appropriate motion primitives: NTOUCHING, R-ALIGN, R-CHASE, TOUCHINGRP, R-AlignRP, R-CHASE, TOUCHIngRP, R-AlignRP, R-CHASE, TOUCHINGRP, $\Lambda$-ALIGNRP, R $\Lambda$-CHASE. 
Now we describe the association of the states with each gap and the landmark. First, $g_{2}^{R}$ is chased, executing states NTouching, R-Align, R-CHASE. Next, $g_{1}^{R}$ is chased, executing states TouchingRP, R-ALIGnRP, R-CHASE. Next, $g_{0}^{R}$ is chased, executing again the states TOUCHINGRP, R-ALIGNRP, R-CHASE. Finally, $\Lambda$ is chased, executing states TouchingRP, $\Lambda$-ALIGnLP, R $\Lambda$-CHASE.

In the previous example all of the gaps in $H$ were of the same type. Using the example illustrated in Figure 7(b), we explain the operation of $M$ when there are different types of gaps. To reach $\Lambda$, the robot chases the sequence $H=\left(g_{1}^{R}, g_{0}^{L}\right)$. The resulting sequence is NTOUCHING, R-ALIGN, R-CHASE (from chasing $g_{1}^{R}$ ), then TOUCHINGRP, L-ALIGNRP, L-CHASE, (from chasing $g_{0}^{L}$ ), and finally TOUCHINGLP, $\Lambda$-ALIGNLP, L $\Lambda$-CHASE (from chasing $\Lambda$ ).

\subsection{Blocked GNT-encoded paths}

We now consider the cases for which either of the first three conditions of Lemma 3 is violated, meaning that the robot would become blocked when applying the GNT in the usual way. For these cases, various forms of "detours" are required. The GNT-encoded path is based on the bitangent lines between intervals in $E$. However, in the configuration space, some bitangent lines disappear. Bitangent lines in the workspace that remain in the configuration space are displaced by a distance $r$ or are rotated by some fixed angle.

The GNT-encoded path cannot be executed by the robot when there is a blockage to chasing $g_{i} \in H$ (or $\Lambda$ ). If this happens it means that: 1) there is no bitangent line between $v_{i+1}$ and $v_{i}$ in $\mathscr{C}, 2$ ) the robot is in a zone in which it cannot detect the crossing of a bitangent line in $\mathscr{C}, 3$ ) there is no clear path to chase $\Lambda$ when the robot sees $\Lambda$, or 4) $\mathscr{C}$ is disconnected. These are the Lemma 3 conditions. We present a solution to deal with the first three cases presented above. However, we do not handle the disconnection of $\mathscr{C}$ because there is no path to $\Lambda$.

If the robot detects a blocked path, then it performs a detour to avoid the obstacles that blocks the GNT-encoded path. We cannot re-plan the entire path to $\Lambda$ because the path depends on the gap $g_{i} \in H$ (or $\Lambda$ ) that is in the gap sensor field of view. For this reason the detour to avoid obstacles is done when the robot detects a blocked path while chasing $g_{i}$ or $\Lambda$.

There are twelve possible cases of blocked paths while chasing a gap $g_{i}$, six of them are related to a right $\operatorname{gap} g_{i}^{R}$ (cases 2, 3, 5, 6, 8 and 9 in Figure 5), and other six totally symmetric cases for a left gap $g_{i}^{L}$. There are only two cases of blocked paths while chasing a landmark $\Lambda$, one occurs when the vertex $u$ representing the subgoal to reach a $\Lambda$ generates a right gap $g^{R}$ and other occurs when the vertex $u$ generates a left gap $g^{L}$. Now, we present the theorem that ensure globally optimal navigation when using $M$.

Theorem 1. The path that the robot center follows when commanded by the automaton $M$, using the information encoded in the GNT and making detours when the path 
to chase $g_{i} \in H$ is blocked or when the path to chase $\Lambda$ is blocked, is optimal in the sense of Euclidean distance.

Proof. The GNT-encoded path is the shortest path for a point in the workspace and it is in the same homotopy class that the shortest path in $\mathscr{C}$ because $E$ and $\mathscr{C}$ are simply connected. We have shown that the sequence of connected intervals in $E$ that the robot traverses is only changed when the conditions of Lemmas 3 are not satisfied; therefore, the shortest path for a disc contains the intervals in $U$. Since each detour is made between consecutive intervals of $U$, and they are locally optimal as proved in Lemma 1 and the path that the disc robot travels whenever the path is not blocked is also locally optimal as proved in Lemma $2^{2}$. Therefore, the resulting global path is optimal.

Constructing a complete GNT: In [18], it has been proved that the construction of the GNT for a point robot will terminate (Lemmas 2 and 3 in [18]). Incompleteness of the GNT is caused by any non-primitive leaves (leaves that correspond to the portions of the environment that have not been perceived by the robot). The key observation to prove the completeness of the GNT for a point robot is that any time that a new gap appears, it must be primitive. If the gap is chased, it cannot split. Therefore, the only gaps that contribute to the incompleteness of the GNT are ones that either appeared in at the beginning of the construction or were formed by a sequence of splits of these gaps. Now, we prove that the construction GNT for a disc robot must also terminate. As mentioned above, compared with the GNT for a point robot, a path that the robot travels to chase a gap will be of two types: nonblocked paths and blocked paths.

Lemma 4. The learning (construction) phase of the GNT for a disc robot always terminates and produces the same GNT as in [18].

Proof. Chasing a gap whenever the path is not blocked is equivalent to chase a gap for a point robot. For blocked path toward a gap, in Lemma $1^{3}$ it has been proved that a blockage to reach a gap can be detected and that the vertex $u_{i}$ that generates the chased gap $g_{i}$ can be reached by traversing detours toward the vertices that blocks the path. Furthermore, while the robot traverses the detours it will always have the information about the identity of the original gap to be chased $g_{i}$, since it is codified in the GNT. Therefore, the construction of the GNT for a disc robot must terminate whenever it terminates for a point robot.

\section{Implementation}

We have implemented the method to further verify its correctness. Figure 8 shows a simulation of the optimal gap navigation for a disc robot. The figure shows snapshots

\footnotetext{
${ }^{2}$ We have also proved other five lemmas for the the remaining cases of a detour toward a right gap and two lemmas for the remaining cases of unblocked path. See [12]

${ }^{3}$ We have also proved other five lemmas for the remaining cases of a detour toward a right gap.
} 


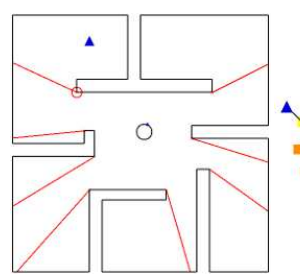

(a)

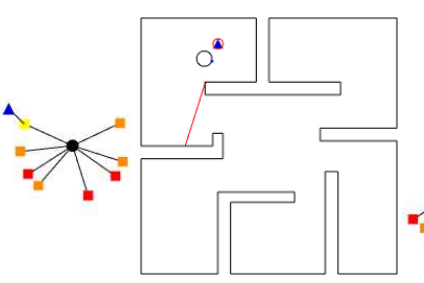

(b) (c)

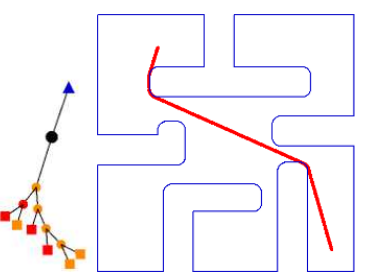

(d)

(e)

Fig. 8 A simulation of optimal gap navigation for a disc robot. Part (e) shows the path in the projected configuration space that the robot traverses to go to the landmark.

of the simulation program. Figures 8 (a) and 8(c) show the robot at different times while following a sequence of gaps to reach the landmark. To the right of each figure is shown the complete GNT with the representation used in [18]. The landmark to be chased is marked as a blue triangle in the workspace and in the GNT as a leaf triangle node. Figure 8(a) shows the robot after the first gap split and the robot is chasing the gap that occludes the landmark. Finally, Figure 8(c) shows the robot chasing the landmark. Figure 8(e) shows the shortest path in the configuration space that the robot traverses to navigate to the landmark. This path was computed based in the information obtained by the robot sensors and using the automaton $M$ from Section 3.

\section{Conclusions}

In this paper we have extended the GNT approach in [18] to a disc-shaped differentialdrive robot. The robot is equipped with simple sensors and it is unable to build precise geometric maps or localize itself in any Euclidean frame. This problem is considerably more challenging than in the case of a point robot because visibility information does not correspond exactly to collision free paths in the configurations space. Consequently, the robot must execute detours from the bitangents in the workspace. Indeed, critical information from the workspace is obtained from the robot's sensors, to infer the optimal robot paths in the configuration space. To solve this problem we developed a motion strategy based on simple sensor feedback and then proved that the motion strategy yields globally optimal motions in the sense of Euclidean distance by characterizing all possible trajectories in terms of sequences of states visited in a finite state machine. Even if precise distance comparisons are not possible, the motion strategy is simple and effective in a broader setting. Important directions for future work include multiply connected environments, disconnected configuration spaces, and bounds respect to optimality for the cases in which all sensing conditions are not met. 
Acknowledgments: This work was supported in part by NSF grants 0904501 (IIS Robotics) and 1035345 (Cyberphysical Systems), DARPA SToMP grant HR001105-1-0008, and MURI/ONR grant N00014-09-1-1052.

\section{References}

1. J. Canny and J. Reif. New lower bound techniques for robot motion planning problems. In Proceedings IEEE Symposium on Foundations of Computer Science, pages 49-60, 1987.

2. D. Z. Chen and H. Wang. Paths among curved obstacles in the plane. In Proceedings of Computing Research Repository, 2011.

3. L. P. Chew. Planning the shortest path for a disc in $O\left(n^{2} \log n\right)$ time. In Proceedings ACM Symposium on Computational Geometry, 1985.

4. M. de Berg, M. van Kreveld, M. Overmars, and O. Schwarzkopf. Computational Geometry: Algorithms and Applications, 2nd Ed. Springer-Verlag, Berlin, 2000.

5. H. Durrant-Whyte and T. Bailey. Simultaneous localization and mapping: Part I. IEEE Robotics and Automation Magazine, 13(2):99-110, 2006.

6. S. K. Ghosh. Visibility Algorithms in the Plane. Cambridge University Press, Cambridge, U.K., 2007.

7. S. K. Ghosh and D. M. Mount. An output sensitive algorithm for computing visibility graphs. In Proceedings IEEE Symposium on Foundations of Computer Science, pages 11-19, 1987.

8. Y. Landa and R. Tsai. Visibility of point clouds and exploratory path planning in unknown environments. Communications in Mathematical Sciences, 6(4):881-913, December 2008.

9. S. M. LaValle. Planning Algorithms. Cambridge University Press, Cambridge, U.K., 2006. Also available at http://planning.cs.uiuc.edu/.

10. S. M. LaValle. Sensing and filtering: A tutorial based on preimages and information spaces. Foundations and Trends in Robotics, 2011. To appear.

11. Yun-Hui Liu and S. Arimoto. Finding the shortest path of a disc among polygonal obstacles using a radius-independent graph. Robotics and Automation, IEEE Trans on, 11(5):682 -691, oct 1995.

12. R. Lopez-Padilla, R. Murrieta-Cid, and S.M. LaValle. Remaining cases of blockage detection and selection of a sub-goal gap. In avaliable on line at: http://www.cimat.mx/\%7Emurrieta/Papersonline/AppendixWAFR12.pdf, pages 1-10, Feb 2012.

13. J. S. B. Mitchell. Shortest paths and networks. In J. E. Goodman and J. O'Rourke, editors, Handbook of Discrete and Computational Geometry, 2nd Ed., pages 607-641. Chapman and Hall/CRC Press, New York, 2004.

14. J. S. B. Mitchell and C. H. Papadimitriou. The weighted region problem. Journal of the ACM, 38:18-73, 1991

15. L. Murphy and P. Newman. Using incomplete online metric maps for topological exploration with the gap navigation tree. In Proc. IEEE Int. Conf. on Robotics \& Automation, 2008.

16. J. H. Reif and Z. Sun. An efficient approximation algorithm for weighted region shortest path problem. In B. R. Donald, K. M. Lynch, and D. Rus, editors, Algorithmic and Computational Robotics: New Directions, pages 191-203. A.K. Peters, Wellesley, MA, 2001.

17. S. Thrun, W. Burgard, and D. Fox. Probabilistic Robotics. MIT Press, Cambridge, MA, 2005.

18. B. Tovar, R Murrieta, and S. M. LaValle. Distance-optimal navigation in an unknown environment without sensing distances. IEEE Trans on Robotics, 23(3):506-518, June 2007. 